\title{
Florística e estrutura da vegetação arbustivo-arbórea em uma área de cerrado rupestre no Parque Estadual da Serra de Caldas Novas, Goiás
}

\author{
Thaís Almeida Lima ${ }^{1,5}$, José Roberto Rodrigues Pinto ${ }^{2}$, Eddie Lenza ${ }^{3}$ \& Alexandre de Siqueira Pinto \\ ${ }^{1}$ Pós-graduação em Ciências de Florestas Tropicais, Instituto Nacional de Pesquisas da Amazônia - INPA, \\ Av. André Araújo, 2936, Aleixo, CEP 69060-001, Manaus, AM, Brasil \\ ${ }^{2}$ Departamento de Engenharia Florestal, Universidade de Brasília - UnB, Brasília, DF, Brasil \\ ${ }^{3}$ Universidade do Estado de Mato Grosso - UNEMAT, Campus de Nova Xavantina, MT, Brasil \\ ${ }^{4}$ Departamento de Ecologia, Universidade de Brasília - UnB, Brasília, DF, Brasil \\ ${ }^{5}$ Autor para correspondência: Thaís Almeida Lima, e-mail: thaisalmeida@inpa.gov.br
}

LIMA, T.A., PINTO, J.R.R., LENZA, E. \& PINTO, A.S. Floristic and structure of woody vegetation of a "cerrado rupestre" area in Serra de Caldas Novas State Park, Goiás. Biota Neotrop. 10(2): http://www. biotaneotropica.org.br/v10n2/en/abstract?article+bn04010022010.

\begin{abstract}
Floristic and structure of woody vegetation of a "cerrado rupestre" area in Serra de Caldas Novas State Park, Goiás): The cerrado stricto sensu is the predominant phytophisiognomy of the Cerrado biome, covering large areas of the Brazilian Central Plateau. When the vegetation grows on rocky soils, it is known locally as "cerrado rupestre". Our knowledge of the tree-shrub communities of this Cerrado vegetation subtype is very limited. The aim of this study was to analyze the floristic composition and structure of tree-shrub vegetation in an area of cerrado rupestre located in the Serra de Caldas Novas State Park (PESCAN), Goiás, Brazil, and to compare the floristic and structural characteristics with those from other areas of cerrado stricto sensu located in Central Brazil. We sampled all individuals with a diameter $\geq 5 \mathrm{~cm}$ at $30 \mathrm{~cm}$ above ground level, presented in 10 randomly plots of $20 \times 50 \mathrm{~m}$. The floristic richness survey revealed 66 species, distributed in 53 genera and 31 botanical families. The studied vegetation had floristic and structural characteristics similar to those recorded in other areas of cerrado stricto sensu growing on deep soils, however, there were some species typically of cerrado rupestre, such as Schwartzia adamantium (Cambess.) Gir--Cañas and Wunderlichia mirabilis Riedel ex Baker and other less abundant characteristic species for this biome, for instance, Peltogyne confertiflora (Mart. ex Hayne) Benth., Myrcia canescens O. Berg and Myrcia variabilis DC. The total basal area was relatively high $\left(12.39 \mathrm{~m}^{2} . \mathrm{ha}^{-1}\right)$ due to the high density of individuals $\left(1.357\right.$ ind.ha $\left.{ }^{-1}\right)$ as well as the abundance of large individuals of species such as Sclerolobium paniculatum Vogel, Pterodon pubescens (Benth.) Benth., S. adamantium and P. confertiflora. Hence, the flat soil surface of cerrado rupestre of PESCAN did not limit the growth of wood vegetation, nor conditioned the establishment of a distinguished flora. Nevertheless, the elevated structural importance of few wood species typical and adapted of rock environments guarantee some floristic peculiarity of the community survey.
\end{abstract}

Keywords: rock outcrops, Central Brazil, phytossociology, savanna.

LIMA, T.A., PINTO, J.R.R., LENZA, E. \& PINTO, A.S. Florística e estrutura da vegetação arbustivo-arbórea em uma área de cerrado rupestre no Parque Estadual da Serra de Caldas Novas, Goiás. Biota Neotrop. 10(2): http://www.biotaneotropica.org.br/v10n2/pt/abstract?article+bn04010022010.

Resumo: (Florística e estrutura da vegetação arbustivo-arbórea em uma área de cerrado rupestre no Parque Estadual da Serra de Caldas Novas, Goiás): O cerrado sentido restrito é a fitofisionomia predominante do bioma Cerrado, ocupando grandes extensões do Planalto Central brasileiro. Quando essa vegetação se desenvolve sobre Neossolos Litólicos recebe a denominação de cerrado rupestre. O conhecimento sobre as comunidades arbustivo-arbóreas deste subtipo fitofisionômico do Cerrado é muito limitado. O objetivo deste estudo foi analisar a composição florística e a estrutura da vegetação arbustivo-arbórea em uma área de cerrado rupestre localizada no Parque Estadual da Serra de Caldas Novas (PESCAN), Goiás, Brasil e comparar suas características florísticas e estruturais com aquelas de outras áreas de cerrado sentido restrito localizadas no Brasil Central. Foram amostrados todos os indivíduos com diâmetro a $30 \mathrm{~cm}$ do solo $\geq 5 \mathrm{~cm}$, presentes em 10 parcelas aleatórias de $20 \times 50 \mathrm{~m}$. A riqueza florística registrada foi de 66 espécies, distribuídas em 53 gêneros e 31 famílias botânicas. A vegetação estudada tem características florísticas e estruturais semelhantes àquelas registradas em outras áreas de cerrado sentido restrito sobre solos profundos, porém, com a presença de algumas espécies típicas de cerrado rupestre, como Schwartzia adamantium (Cambess.) Gir.-Cañas e Wunderlichia mirabilis Riedel ex Baker e algumas raras para o bioma, por exemplo, Peltogyne confertiflora (Mart. ex Hayne) Benth., Myrcia canescens O. Berg e Myrcia variabilis DC. A área basal encontrada foi relativamente elevada $\left(12,39 \mathrm{~m}^{2} . \mathrm{ha}^{-1}\right)$ devido à alta densidade de indivíduos (1.357 ind.ha $\left.{ }^{-1}\right)$, bem como a abundância de indivíduos de grande porte, de espécies como Sclerolobium paniculatum Vogel, Pterodon pubescens (Benth.) Benth., S. adamantium e P. confertiflora. Dessa forma, o substrato raso sob o cerrado rupestre do PESCAN, não limitou o desenvolvimento da vegetação arbustivo-arbórea, nem condicionou o estabelecimento de uma flora diferenciada. No entanto, a elevada importância estrutural de poucas espécies lenhosas típicas e adaptadas aos ambientes rupestres garante certa peculiaridade florística à comunidade estudada.

Palavras-chave: afloramentos rochosos, Brasil Central, fitossociologia, savana. 


\section{Introdução}

O Cerrado é o segundo maior e um dos mais diversos biomas do Brasil, ocupa aproximadamente $21 \%$ do território nacional e abriga cerca de 33\% da diversidade biológica brasileira (Aguiar et al. 2004). No entanto, o modelo de ocupação implantado na região Centro-Oeste, com a conversão de grandes extensões de vegetação nativa em pastagens e monoculturas contribuiu para que o Cerrado se tornasse um dos biomas mais ameaçados do mundo (Klink \& Machado 2005). Estima-se que a área remanescente de vegetação nativa do Cerrado é de aproximadamente $46,74 \%$, baseado em imagens de satélite de 2002 (MMA 2007). A grande preocupação que se tem com este modelo de ocupação se deve ao fato do Cerrado ser a savana floristicamente mais rica do mundo (Walter 2006). A elevada riqueza de espécies, o alto grau de endemismo e a intensa destruição de habitats colocam o Cerrado na lista de hotspots mundiais, ou seja, áreas prioritárias à conservação da biodiversidade (Mittermeier et al. 2005).

A vegetação do Cerrado é composta por um mosaico representado por formações florestais, savânicas e campestres (Ribeiro \& Walter 2008). As formações savânicas de interflúvio, principalmente o cerrado sentido restrito, dominam a paisagem do Planalto Central brasileiro (Felfili et al. 2005). De acordo com Ribeiro \& Walter (2008) o cerrado sentido restrito pode ser subdividido em quatro subtipos fitofisionômicos, ou seja, cerrado denso, típico, ralo e rupestre. Os três primeiros são diferenciados pela densidade do estrato lenhoso, sendo esta crescente do cerrado ralo ao denso, enquanto o cerrado rupestre ocorre em solos rasos e com afloramentos rochosos, denominados de ambientes rupestres. Nesses ambientes a vegetação lenhosa se estabelece nas fendas e nos degraus formados entre as rochas onde há acumulo de solo e microsítios para o estabelecimento das plantas (Ribeiro \& Walter 2008, Giulietti et al. 2000).

A flora dos ambientes rupestres apresenta elevada riqueza e alto grau de endemismo (Harley 1995, Oliveira-Filho \& Fluminhan-Filho 1999), além de inúmeras espécies raras, principalmente no componente herbáceo-arbustivo (Pirani et al. 1994, Romero \& Nakajima 1999, Romero \& Martins 2002, Vitta 2002). Se considerarmos que estas áreas ocupam aproximadamente $7 \%$ da área de domínio do Cerrado (Reatto et al. 1998) e que parte da vegetação nativa remanescente e bem preservada está localizada nestes locais (Machado et al. 2004), é premente a necessidade de estudos sobre a vegetação arbustivoarbórea nas diferentes regiões de ocorrência do cerrado rupestre.

O presente estudo tem como objetivo analisar a composição florística e a estrutura da vegetação arbustivo-arbórea em uma área de cerrado rupestre localizada no Parque Estadual da Serra de Caldas Novas (GO) e avaliar as semelhanças florísticas e estruturais entre a vegetação estudada e a de outros levantamentos realizados em cerrado sentido restrito localizados no Brasil Central.

\section{Material e Métodos}

\section{Local de estudo}

O estudo foi realizado em uma área de cerrado rupestre (Figura 1) localizada no Parque Estadual da Serra de Caldas Novas - PESCAN, Estado de Goiás (17 44' 40,8” S e 48 39' 49,6” O). O PESCAN possui uma área com cerca de 12.500 ha e está situado entre os municípios de Caldas Novas e Rio Quente (Agência Ambiental de Goiás 2009). O clima da região é do tipo Aw, segundo o sistema de

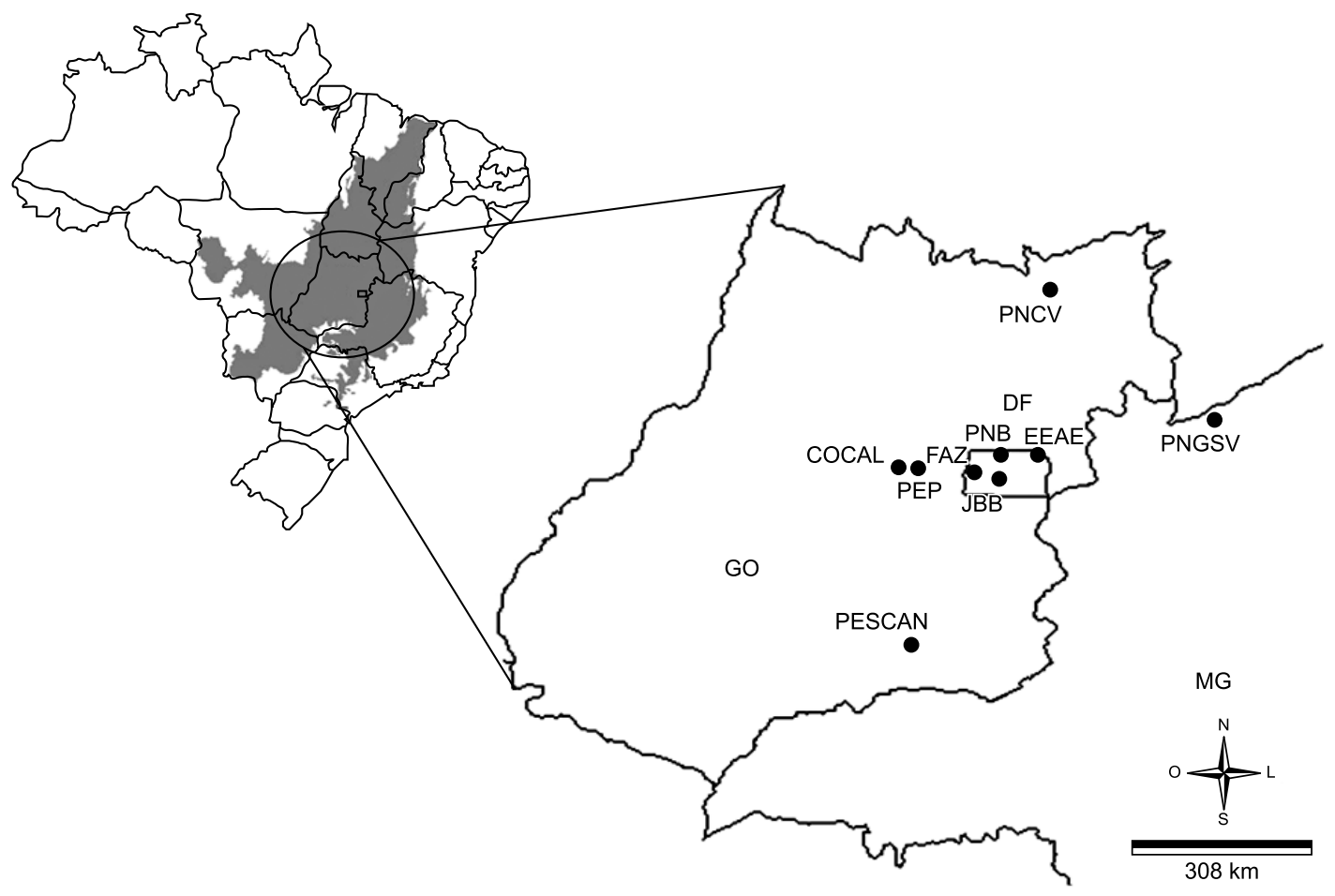

Figura 1. Localização da área de cerrado rupestre estudada no Parque Estadual da Serra de Caldas Novas (PESCAN), Goiás e as áreas usadas para comparação no presente estudo. Em destaque no mapa do Brasil a área de distribuição do bioma Cerrado. Onde, COCAL: Cocalzinho de Goiás, PEP: Parque Estadual dos Pireneus, PNB: Parque Nacional de Brasília, EEAE: Estação Ecológica das Águas Emendadas, FAZ: Fazenda Sucupira, JBB: Jardim Botânico de Brasília, PNGSV: Parque Nacional do Grande Sertão Veredas, PNCV: Parque Nacional da Chapada dos Veadeiros.

Figure 1. Location of the "cerrado rupestre" area surveyed in the Serra de Caldas Novas State Park (PESCAN), Goiás and areas used for comparison in the present study. From the map of the distribution of the Cerrado biome within Brazil. Where, COCAL: Cocalzinho de Goiás, PEP: Pireneus State Park, PNB: Brasília National Park, EEAE: Ecological Station of Águas Emendadas, FAZ: Sucupira Farm, JBB: Brasília Botanical Garden, PNGSV: Grande Sertão Veredas National Park, PNCV: Chapada dos Veadeiros National Park. 
classificação de Köppen, apresentando pluviosidade média anual de $1.500 \mathrm{~mm}$, com as chuvas concentrando-se no período de outubro a março (Nimer 1989).

Na Serra de Caldas Novas ocorre um mosaico de formas vegetacionais associado a um gradiente topográfico, variando desde formações florestais, como cerradão e matas de galeria, até formações campestres, como campo rupestre (Eiten 1978). Na base e no topo do platô, os solos são profundos podendo ser classificados como Latossolos e Neossolos Quartzarênicos, e tem como vegetação predominante o cerrado típico. Nas encostas onde ocorrem Cambissolos e Neossolos Litólicos, associados aos afloramentos rochosos, a vegetação é constituída por campos rupestres e cerrado rupestre (observações de campo).

\section{Amostragem da vegetação}

Foram alocadas 10 parcelas de área fixa de $20 \times 50 \mathrm{~m}$, conforme utilizado para levantamentos da vegetação nas formações savânicas do bioma Cerrado (Felfili et al. 2001). As parcelas foram alocadas nas áreas de afloramentos rochosos, distribuídas com distância mínima de 150 m e máxima de $1.700 \mathrm{~m}$. A maior extensão das parcelas foi estabelecida ao longo do gradiente topográfico, com a declividade do terreno ajustada por meio do nivelamento da trena, a fim de corrigir a distância horizontal, conforme sugerido por Oliveira-Filho (1994).

Todos os indivíduos arbustivo-arbóreos vivos, incluindo as monocotiledôneas, com DAS (diâmetro a altura de $30 \mathrm{~cm}$ do solo) $\geq 5 \mathrm{~cm}$ encontrados no interior das parcelas foram registrados e identificados ao nível de espécie. Para os indivíduos não identificados no campo foram coletadas amostras de material botânico e herborizadas para posterior identificação, através de consultas a especialistas e comparação com o acervo do Herbário da Universidade de Brasília (Herbário UB). Mediu-se o diâmetro (DAS) e a altura total, conforme metodologia adotada para levantamentos da vegetação arbustivo-arbórea em áreas de cerrado sentido restrito (Felfili et al.
2001). Nos casos de indivíduos que apresentaram ramificações abaixo de $30 \mathrm{~cm}$, para o cálculo da área basal, foi calculada a soma da área seccional de cada ramificação. O sistema de classificação botânica adotado foi o "Angiosperm Phylogeny Group" (APG II 2003). O nome científico das espécies foi verificado junto ao banco de dados do Missouri Botanical Garden (http://www.tropicos.org).

\section{Análises dos dados}

A estrutura da comunidade foi descrita com base nos parâmetros fitossociológicos de acordo com Müeller-Dombois \& Ellenberg (1974) e na distribuição de frequência dos indivíduos nas classes de diâmetros e de altura (Scolforo 1993). Os parâmetros fitossociológicos e os índices de diversidade de Shannon (H') e de Pielou (J') foram calculados com o uso do Programa Mata Nativa 2 (CIENTEC 2006). Com o auxílio do programa EstimateS 8.0 (Colwell 2006), estimou-se a riqueza da comunidade por meio do estimador Chaol (Chao 1984).

A similaridade florística entre a área do presente estudo e outras áreas de cerrado rupestre e cerrado sentido restrito sobre solos profundos localizadas no Brasil Central, foi calculada pelo índice de Czekanowski ( $\mathrm{S}_{\mathrm{C}}$ ) (Kent \& Coker 1992). Esse índice, dado em valores percentuais, apresenta elevada acurácia na determinação da similaridade entre comunidades (Bloom 1981) e indica alta similaridade quando apresenta valores superiores a $50 \%$ (Kent \& Coker 1992). As comparações foram realizadas preferencialmente com estudos desenvolvidos em Unidades de Conservação e com metodologia de amostragem similar à utilizada no presente estudo.

\section{Resultados}

Foram registradas 66 espécies, distribuídas em 53 gêneros e 31 famílias botânicas (Tabela 1). Pelo índice de Chao1, a riqueza estimada foi 89,14 espécies, com mínimo e máximo de 73,35 e 138,86 espécies, respectivamente. A densidade encontrada foi de

Tabela 1. Parâmetros fitossociológicos, em ordem decrescente do valor de importância (VI), para as espécies arbustivo-arbóreas (diâmetro a 30 cm do solo $\geq 5 \mathrm{~cm}$ ) amostradas em 10 parcelas de $20 \times 50 \mathrm{~m}$ em uma área de cerrado rupestre no Parque Estadual da Serra de Caldas Novas, GO. Onde: DA = densidade absoluta (ind.ha $\left.{ }^{-1}\right), \mathrm{DR}=$ densidade relativa $(\%), \mathrm{FA}=$ frequência absoluta $(\%$ de parcelas onde ocorre a espécie $), \mathrm{FR}=$ frequência relativa $(\%)$, DoA $=$ dominância absoluta $\left(\mathrm{m}^{2} \cdot \mathrm{ha}^{-1}\right)$ e DoR $=$ dominância relativa $(\%)$.

Table 1. Phytossociological parameters, ordained by decreasing VI, for woody species (diameter $\geq 5 \mathrm{~cm}$ at $30 \mathrm{~cm}$ above ground level) sampled in 10 plots of $20 \times 50 \mathrm{~m}$ in an area of the "cerrado rupestre" of Serra de Caldas Novas State Park, GO. DA $=$ absolute density $\left(\right.$ ind.ha $\left.{ }^{-1}\right), \mathrm{DR}=$ relative density $(\%)$, FA $=$ ab-

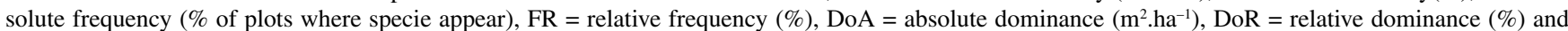
$\mathrm{VI}=$ importance value

\begin{tabular}{|c|c|c|c|c|c|c|c|c|}
\hline Espécie & Família & DA & DR & FA & FR & DoA & DoR & VI \\
\hline Sclerolobium paniculatum Vogel & Fabaceae & 137 & 10,10 & 100 & 3,51 & 3,174 & 25,61 & 39,21 \\
\hline Peltogyne confertiflora (Mart. ex Hayne) Benth. & Fabaceae & 149 & 10,98 & 90 & 3,16 & 0,818 & 6,60 & 20,74 \\
\hline Pterodon pubescens (Benth.) Benth. & Fabaceae & 72 & 5,31 & 100 & 3,51 & 0,888 & 7,17 & 15,98 \\
\hline Schwartzia adamantium (Cambess.) Gir.-Cañas & Marcgraviaceae & 47 & 3,46 & 100 & 3,51 & 0,875 & 7,06 & 14,04 \\
\hline Caryocar brasiliense Cambess. & Caryocaraceae & 75 & 5,53 & 90 & 3,16 & 0,633 & 5,11 & 13,79 \\
\hline Wunderlichia mirabilis Riedel ex Baker & Asteraceae & 54 & 3,98 & 100 & 3,51 & 0,768 & 6,19 & 13,68 \\
\hline Qualea multiflora Mart. & Vochysiaceae & 77 & 5,67 & 100 & 3,51 & 0,446 & 3,60 & 12,78 \\
\hline Lafoensia pacari A. St.-Hil. & Lythraceae & 75 & 5,53 & 100 & 3,51 & 0,464 & 3,74 & 12,78 \\
\hline Plathymenia reticulata Benth. & Fabaceae & 54 & 3,98 & 100 & 3,51 & 0,516 & 4,16 & 11,65 \\
\hline Dalbergia miscolobium Benth. & Fabaceae & 60 & 4,42 & 60 & 2,11 & 0,361 & 2,91 & 9,44 \\
\hline Salacia crassifolia (Mart. ex Schult.) G. Don & Hippocrateaceae & 56 & 4,13 & 100 & 3,51 & 0,201 & 1,62 & 9,25 \\
\hline Kielmeyera coriacea Mart. \& Zucc. & Clusiaceae & 50 & 3,68 & 100 & 3,51 & 0,233 & 1,88 & 9,07 \\
\hline Qualea parviflora Mart. & Vochysiaceae & 40 & 2,95 & 70 & 2,46 & 0,454 & 3,66 & 9,07 \\
\hline Plenckia populnea Reissek & Celastraceae & 43 & 3,17 & 100 & 3,51 & 0,212 & 1,71 & 8,39 \\
\hline Aspidosperma macrocarpon Mart. & Apocynaceae & 32 & 2,36 & 90 & 3,16 & 0,313 & 2,53 & 8,04 \\
\hline Aspidosperma tomentosum Mart. & Apocynaceae & 39 & 2,87 & 100 & 3,51 & 0,202 & 1,63 & 8,02 \\
\hline
\end{tabular}


Tabela 1. Continuação...

\begin{tabular}{|c|c|c|c|c|c|c|c|c|}
\hline Espécie & Família & DA & DR & FA & FR & DoA & DoR & VI \\
\hline Psidium myrsinoides $\mathrm{O}$. Berg & Myrtaceae & 40 & 2,95 & 90 & 3,16 & 0,174 & 1,40 & 7,51 \\
\hline Ouratea hexasperma (A. St.-Hil.) Baill. & Ochnaceae & 35 & 2,58 & 70 & 2,46 & 0,198 & 1,60 & 6,63 \\
\hline Vatairea macrocarpa (Benth.) Ducke & Fabaceae & 25 & 1,84 & 70 & 2,46 & 0,202 & 1,63 & 5,93 \\
\hline Qualea grandiflora Mart. & Vochysiaceae & 19 & 1,4 & 60 & 2,11 & 0,166 & 1,34 & 4,84 \\
\hline Hymenaea stigonocarpa Mart. ex Hayne & Fabaceae & 15 & 1,11 & 60 & 2,11 & 0,148 & 1,20 & 4,41 \\
\hline Hancornia speciosa B.A. Gomes & Apocynaceae & 12 & 0,88 & 50 & 1,75 & 0,067 & 0,54 & 3,18 \\
\hline Connarus suberosus Planch. & Connaraceae & 11 & 0,81 & 50 & 1,75 & 0,041 & 0,33 & 2,90 \\
\hline Acosmium dasycarpum (Vogel) Yakovlev & Fabaceae & 8 & 0,59 & 40 & 1,4 & 0,103 & 0,83 & 2,82 \\
\hline Kielmeyera speciosa A. St.-Hil. & Clusiaceae & 8 & 0,59 & 50 & 1,75 & 0,045 & 0,36 & 2,71 \\
\hline Andira paniculata Benth. & Fabaceae & 11 & 0,81 & 40 & 1,4 & 0,038 & 0,31 & 2,52 \\
\hline Guapira noxia (Netto) Lundell & Nyctaginaceae & 8 & 0,59 & 40 & 1,4 & 0,058 & 0,47 & 2,46 \\
\hline Neea theifera Oerst. & Nyctaginaceae & 10 & 0,74 & 40 & 1,4 & 0,033 & 0,26 & 2,40 \\
\hline Myrcia canescens O. Berg & Myrtaceae & 6 & 0,44 & 50 & 1,75 & 0,018 & 0,15 & 2,35 \\
\hline Pseudobombax longiflorum (Mart. \& Zucc.) A. Robyns & Malvaceae & 6 & 0,44 & 40 & 1,4 & 0,042 & 0,34 & 2,18 \\
\hline Pouteria ramiflora (Mart.) Radlk. & Sapotaceae & 6 & 0,44 & 30 & 1,05 & 0,079 & 0,64 & 2,13 \\
\hline Machaerium opacum Vogel & Fabaceae & 5 & 0,37 & 40 & 1,4 & 0,026 & 0,21 & 1,98 \\
\hline Byrsonima coccolobifolia Kunth & Malpighiaceae & 5 & 0,37 & 40 & 1,4 & 0,020 & 0,16 & 1,93 \\
\hline Palicourea rigida Kunth & Rubiaceae & 7 & 0,52 & 30 & 1,05 & 0,037 & 0,30 & 1,87 \\
\hline Schefflera macrocarpa (Cham. \& Schltdl.) Frodin & Araliaceae & 4 & 0,29 & 30 & 1,05 & 0,051 & 0,41 & 1,76 \\
\hline Myrcia variabilis DC. & Myrtaceae & 6 & 0,44 & 30 & 1,05 & 0,018 & 0,14 & 1,64 \\
\hline Tabebuia serratifolia (Vahl) G. Nicholson & Bignoniaceae & 5 & 0,37 & 20 & 0,7 & 0,065 & 0,53 & 1,60 \\
\hline Annona coriacea Mart. & Annonaceae & 4 & 0,29 & 30 & 1,05 & 0,028 & 0,22 & 1,57 \\
\hline Davilla elliptica A. St.-Hil. & Dilleniaceae & 3 & 0,22 & 30 & 1,05 & 0,009 & 0,07 & 1,35 \\
\hline Erythroxylum tortuosum Mart. & Erythroxylaceae & 3 & 0,22 & 20 & 0,7 & 0,007 & 0,05 & 0,98 \\
\hline Brosimum gaudichaudii Trécul & Moraceae & 2 & 0,15 & 20 & 0,7 & 0,012 & 0,10 & 0,95 \\
\hline Byrsonima pachyphylla A. Juss. & Malpighiaceae & 2 & 0,15 & 20 & 0,7 & 0,008 & 0,07 & 0,92 \\
\hline Guapira graciliflora (Schmidt) Lundell & Nyctaginaceae & 2 & 0,15 & 20 & 0,7 & 0,006 & 0,05 & 0,90 \\
\hline Chomelia ribesioides Benth. ex A. Gray & Rubiaceae & 2 & 0,15 & 20 & 0,7 & 0,004 & 0,04 & 0,89 \\
\hline Maprounea guianensis Aubl. & Euphorbiaceae & 3 & 0,22 & 10 & 0,35 & 0,018 & 0,15 & 0,72 \\
\hline Syagrus flexuosa (Mart.) Becc. & Arecaceae & 2 & 0,15 & 10 & 0,35 & 0,017 & 0,14 & 0,64 \\
\hline Eremanthus glomerulatus Less. & Asteraceae & 2 & 0,15 & 10 & 0,35 & 0,008 & 0,07 & 0,56 \\
\hline Tabebuia ochracea (Cham.) Standl. & Bignoniaceae & 2 & 0,15 & 10 & 0,35 & 0,007 & 0,05 & 0,55 \\
\hline Eriotheca pubescens (Mart. \& Zucc.) Schott \& Endl. & Malvaceae & 1 & 0,07 & 10 & 0,35 & 0,011 & 0,09 & 0,52 \\
\hline Miconia ferruginata DC. & Melastomataceae & 1 & 0,07 & 10 & 0,35 & 0,009 & 0,07 & 0,50 \\
\hline Eriotheca gracilipes (K. Schum.) A. Robyns & Malvaceae & 1 & 0,07 & 10 & 0,35 & 0,008 & 0,07 & 0,49 \\
\hline Eugenia involucrata DC. & Myrtaceae & 1 & 0,07 & 10 & 0,35 & 0,007 & 0,05 & 0,48 \\
\hline Xylopia aromatica (Lam.) Mart. & Annonaceae & 1 & 0,07 & 10 & 0,35 & 0,005 & 0,04 & 0,47 \\
\hline Miconia albicans (Sw.) Triana & Melastomataceae & 1 & 0,07 & 10 & 0,35 & 0,005 & 0,04 & 0,46 \\
\hline Vochysia rufa Mart. & Vochysiaceae & 1 & 0,07 & 10 & 0,35 & 0,004 & 0,03 & 0,46 \\
\hline Bowdichia virgilioides Kunth & Fabaceae & 1 & 0,07 & 10 & 0,35 & 0,004 & 0,03 & 0,46 \\
\hline Tocoyena formosa (Cham. \& Schltdl.) K. Schum. & Rubiaceae & 1 & 0,07 & 10 & 0,35 & 0,004 & 0,03 & 0,46 \\
\hline Licania humilis Cham. \& Schltdl. & Chrysobalanaceae & 1 & 0,07 & 10 & 0,35 & 0,004 & 0,03 & 0,46 \\
\hline Simarouba versicolor A. St.-Hil. & Simaroubaceae & 1 & 0,07 & 10 & 0,35 & 0,004 & 0,03 & 0,45 \\
\hline Casearia sylvestris $\mathrm{Sw}$. & Salicaceae & 1 & 0,07 & 10 & 0,35 & 0,003 & 0,03 & 0,45 \\
\hline Myrcia linguaeformis (Berg) N. Silveira & Myrtaceae & 1 & 0,07 & 10 & 0,35 & 0,003 & 0,03 & 0,45 \\
\hline Aegiphila lhotskiana Cham. & Verbenaceae & 1 & 0,07 & 10 & 0,35 & 0,003 & 0,02 & 0,45 \\
\hline Cordiera elliptica (Cham.) O. Kuntze & Rubiaceae & 1 & 0,07 & 10 & 0,35 & 0,003 & 0,02 & 0,45 \\
\hline Aspidosperma ramiflorum Müll. Arg. & Apocynaceae & 1 & 0,07 & 10 & 0,35 & 0,002 & 0,02 & 0,44 \\
\hline Stryphnodendron obovatum Benth. & Fabaceae & 1 & 0,07 & 10 & 0,35 & 0,002 & 0,02 & 0,44 \\
\hline Asteraceae sp. 1 & Asteraceae & 1 & 0,07 & 10 & 0,35 & 0,002 & 0,02 & 0,44 \\
\hline Total & & 1.357 & 100 & 2.850 & 100 & 12,394 & 100,00 & 300 \\
\hline
\end{tabular}


1.357 ind.ha ${ }^{-1} \mathrm{e}$ a área basal $12,39 \mathrm{~m}^{2} . \mathrm{ha}^{-1}$. O índice de diversidade de Shannon (H') foi 3,331 nats.ind ${ }^{-1}$ e o índice de eqüabilidade de Pielou (J') foi 0,795 .

As cinco famílias com maior número de espécies foram Fabaceae (12), Myrtaceae (5), Apocynaceae (4), Rubiaceae (4) e Vochysiaceae (4). Por outro lado, 19 famílias (59,38\% do total) foram registradas na área com apenas uma espécie cada. Analisando as famílias em termos de abundância de indivíduos, quinze delas foram amostradas com 10 ou mais indivíduos, juntas elas representam 95,80\% da densidade total e $65,15 \%$ da riqueza de espécies registrada na área. Quatro famílias (12,09\% do total) foram representadas por apenas um indivíduo: Chrysobalanaceae (Licania humilis Cham. \& Schltdl.), Salicaceae (Casearia sylvestris Sw.), Simaroubaceae (Simarouba versicolor A. St.-Hil.) e Verbenaceae (Aegiphila lhotskiana Cham.).

As 10 espécies com maiores valores de importância foram, respectivamente: Sclerolobium paniculatum Vogel, Peltogyne confertiflora (Mart. ex Hayne) Benth., Pterodon pubescens (Benth.) Benth., Schwartzia adamantium (Cambess.) Gir.-Cañas, Caryocar brasiliense Cambess., Wunderlichia mirabilis Riedel ex Baker, Qualea multiflora Mart., Lafoensia pacari A. St.-Hil., Plathymenia reticulata Benth. e Dalbergia miscolobium Benth. Juntas elas correspondem a $58,95 \%$ da densidade absoluta e $54,69 \%$ do VI total da comunidade. Dezoito espécies, $27,27 \%$ do total da comunidade, foram consideradas localmente raras, ou seja, com um indivíduo por hectare (Martins 1991) e representaram 1,32\% dos indivíduos amostrados e $2,77 \%$ do VI total.

A distribuição de frequência dos diâmetros (Figura 2) seguiu o padrão de distribuição em "J-reverso" ou exponencial negativa, com a maioria dos indivíduos concentrados nas três primeiras classes de diâmetro, somando $72,66 \%$ do total de indivíduos amostrados. Apenas seis espécies, Acosmium dasycarpum (Vogel) Yakovlev, Dalbergia miscolobium, Pterodon pubescens, Schwartzia adamantium, Sclerolobium paniculatum e Wunderlichia mirabilis apresentaram indivíduos com diâmetros superiores a $21 \mathrm{~cm}$. Com relação à distribuição de alturas (Figura 3), 81,42\% dos indivíduos possuem altura de até 4,2 m e oito espécies (Aspidosperma macrocarpon Mart., Eriotheca

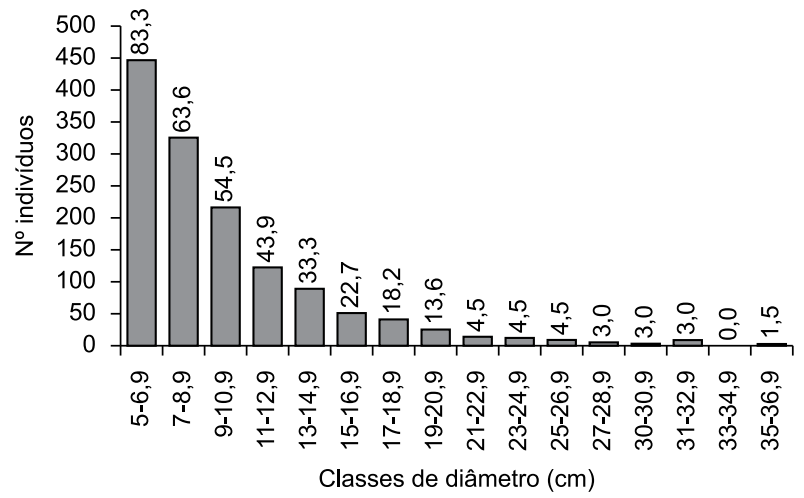

Figura 2. Distribuição de frequência nas classes de diâmetro dos indivíduos arbustivo-arbóreos (diâmetro a $30 \mathrm{~cm}$ do solo $\geq 5 \mathrm{~cm}$ ) amostrados em 10 parcelas de $20 \times 50 \mathrm{~m}$, em uma área de cerrado rupestre do Parque Estadual da Serra de Caldas Novas, GO. Os números acima das barras indicam a porcentagem de espécies por classe.

Figure 2. Frequency distribution in the diameter class of the individuals of woody species (diameter $\geq 5 \mathrm{~cm}$ at $30 \mathrm{~cm}$ above ground level) sampled in 10 plots of $20 \times 50 \mathrm{~m}$ in an area of the "cerrado rupestre" of Serra de Caldas Novas State Park, GO. The numbers above the bars indicate the species percentage by size class. pubescens (Mart. \& Zucc.) Schott \& Endl., Hymenaea stigonocarpa Mart. ex Hayne, Pterodon pubescens, Qualea multiflora, Sclerolobium paniculatum, Tabebuia serratifolia (Vahl) G. Nicholson e Wunderlichia mirabilis) foram representadas por indivíduos com alturas superiores a 5,8 m. Essas espécies compõem o estrato superior da vegetação, do qual Sclerolobium paniculatum se destaca como espécie emergente, por ser a única cujos indivíduos alcançaram alturas superiores a $9 \mathrm{~m}$.

A similaridade florística entre a área amostrada e as outras áreas de cerrado rupestre e cerrado sentido restrito usadas nas comparações apresentou baixos valores e sem tendências evidentes para maiores similaridades entre as áreas de cerrado rupestre comparadas (Tabela 2). A maior similaridade encontrada $\left(S_{C}=30,76\right)$ foi com a área de cerrado sentido restrito sobre solo profundo amostrado na Estação Ecológica de Águas Emendadas (DF) (Felfili et al. 1994). Por outro lado, a menor similaridade florística $\left(S_{C}=17,49\right)$ foi verificada com uma das áreas de cerrado rupestre amostrada no Parque Estadual dos Pireneus (GO) (Moura 2006). De maneira semelhante, os valores de riqueza e diversidade de espécies, densidade de indivíduos e área basal da comunidade, também não apresentaram tendências evidentes para valores mais próximos entre comunidades com substrato semelhante (Tabela 2).

\section{Discussão}

A riqueza arbustivo-arbórea registrada em 1,0 ha de cerrado rupestre no PESCAN (66 espécies) foi semelhante àquela encontrada em outros estudos de cerrado rupestre no Brasil Central, adotando a mesma metodologia, pois está dentro dos limites encontrados naqueles estudos (51 a 81 espécies) (Tabela 2). A riqueza total estimada pelo índice de Chao1 (89 espécies) foi bem superior àquela observada, isto se deve ao fato de que esse índice é fortemente influenciado por espécies com somente uma ocorrência (Santos 2003) e na comunidade estudada cerca de um quarto das espécies foram representadas por um só indivíduo. Apenas uma área de cerrado sentido restrito sobre afloramentos rochosos no nordeste goiano e sul do Tocantins (Felfili \& Fagg 2007) apresentou maior riqueza (87 espécies), provavelmente porque foram incluídos neste estudo

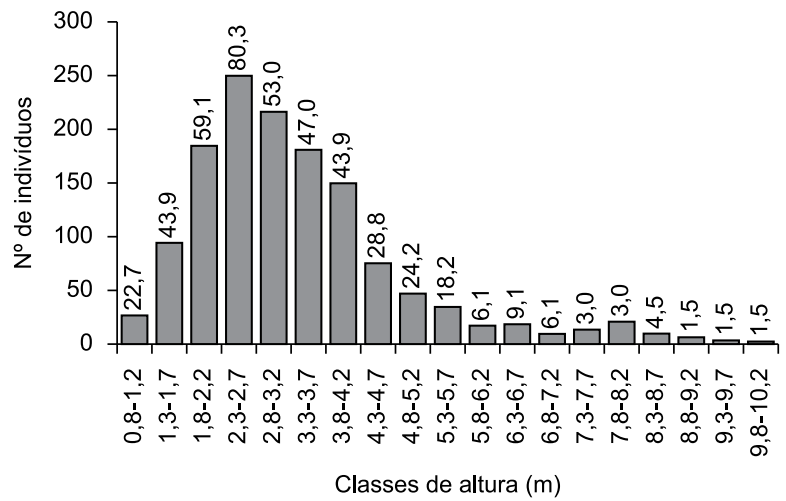

Figura 3. Distribuição de frequência nas classes de altura dos indivíduos arbustivo-arbóreos (diâmetro a $30 \mathrm{~cm}$ do solo $\geq 5 \mathrm{~cm}$ ) amostrados em 10 parcelas de $20 \times 50 \mathrm{~m}$, em uma área de cerrado rupestre do Parque Estadual da Serra de Caldas Novas, GO. Os números acima das barras indicam a porcentagem de espécies por classe.

Figure 3. Frequency distribution in the height class of the individuals of woody species (diameter $\geq 5 \mathrm{~cm}$ at $30 \mathrm{~cm}$ above ground level) sampled in 10 plots of $20 \times 50 \mathrm{~m}$ in an area of the "cerrado rupestre" of Serra de Caldas Novas State Park, GO. The numbers above the bars indicate the species percentage by size class. 
Tabela 2. Riqueza de espécies (S), densidade absoluta (DA), área basal (AB), índice de diversidade Shannon (H'), índice de equabilidade de Pielou (J) e índice de similaridade de Czekanowski $\left(\mathrm{S}_{\mathrm{C}}\right)$ em áreas de cerrado rupestre e cerrado sentido restrito sobre solos profundos do Brasil Central.

Table 2. Species richness (S), absolute density (DA), basal area (AB), Shannon diversity index (H'), Pielou evenness index (J) and Czekanowski similarity index $\left(\mathrm{S}_{\mathrm{C}}\right)$ in cerrado rupestre and cerrado strictu sensu on deep soils areas of Brasil Central.

\begin{tabular}{|c|c|c|c|c|c|c|c|c|}
\hline Local* & Tipo de Solo & $\mathbf{S}$ & $\begin{array}{c}\text { DA } \\
\left(\text { ind.ha }^{-1}\right)\end{array}$ & $\begin{array}{c}\mathbf{A B} \\
\left(\mathbf{m}^{2} \cdot \mathbf{h a}^{-1}\right)\end{array}$ & $\begin{array}{c}\mathbf{H}^{\prime} \\
\left.\text { (nats.ind }^{-1}\right)\end{array}$ & $\mathbf{J}$ & $\begin{array}{l}\mathrm{S}_{\mathrm{C}} \\
(\%)\end{array}$ & Autor \\
\hline \multicolumn{9}{|l|}{ Cerrado rupestre } \\
\hline Parque Estadual da Serra de Caldas Novas (GO) & Neossolo Litólico & 66 & 1.357 & 12,39 & 3,331 & 0,790 & - & Presente Estudo \\
\hline Parque Estadual dos Pireneus (GO) Área 1 & Neossolo Litólico & 65 & 1.105 & 11,03 & 3,650 & 0,870 & 20,43 & Moura (2006) \\
\hline Parque Estadual dos Pireneus (GO) Área 2 & Neossolo Litólico & 56 & 507 & 3,91 & 3,33 & 0,820 & 17.49 & Moura (2006) \\
\hline Fazenda Sucupira (DF) & Neossolo Litólico & 51 & 631 & 3,67 & 3,087 & - & 25,25 & Amaral et al. (2006) \\
\hline Cocalzinho de Goiás (GO) & Neossolo Litólico & 65 & 674 & 5,67 & 3,450 & 0,827 & 27,47 & Pinto et al. (2009) \\
\hline Parque Nacional da Chapada dos Veadeiros (GO) & Neossolo Litólico & 81 & 1.109 & 8,92 & 3,491 & 0,806 & 21,71 & Felfili et al. (2007) \\
\hline \multicolumn{9}{|l|}{ Cerrado sentido restrito sobre solos profundos } \\
\hline Estação Ecológica de Águas Emendadas (DF) & Latossolo & 72 & 1.396 & 10,76 & 3,620 & 0,846 & 30,76 & Felfili et al. (1994) \\
\hline Jardim Botânico de Brasília (DF) & Latossolo & 53 & 1.219 & 8,60 & 3,160 & - & 24,65 & Fonseca \& Silva Jr. (2004) \\
\hline Parque Nacional de Brasília (DF) & Latossolo & 55 & 1036 & 10,76 & 3,419 & 0,853 & 25,42 & Felfili et al. (1994) \\
\hline Parque Nacional Grande Sertão Veredas (MG) & Neossolo Quartzarênico & 67 & 825 & 8,89 & 3,442 & 0,810 & 29,22 & Felfili et al. (2001) \\
\hline
\end{tabular}

*Todos os trabalhos usados para comparação amostraram 1 ha (10 parcelas de $20 \times 50 \mathrm{~m}$ ), com diâmetro a $30 \mathrm{~cm}$ do solo $\geq 5 \mathrm{~cm}$, como critério de inclusão.

* All surveys used for comparison sampled 1 ha $(10$ plots of $20 \times 50 \mathrm{~m})$, with diameter $\geq 5 \mathrm{~cm}$ at $30 \mathrm{~cm}$ above ground level, as inclusion standard.

sítios geograficamente distantes e, em certos casos, sobre diferentes tipos de solo, como Neossolos Litólicos, Cambissolos e Plintossolos com concreções lateríticas. Além disso, a região da Chapada dos Veadeiros, incluída no referido estudo, é conhecida como um dos centros de endemismo e por apresentar uma das maiores riquezas de espécies lenhosas do Cerrado (Munhoz \& Proença 1998, Simon \& Proença 2000, Mendonça et al. 2007).

De maneira semelhante, a riqueza arbustivo-arbórea do cerrado rupestre registrada no PESCAN é similar àquela de áreas de cerrado sentido restrito sobre solos profundos (53 a 72 espécies) (Tabela 2). A riqueza registrada no presente estudo foi próxima das 67 espécies encontradas em um levantamento, adotando método de ponto quadrante, também conduzido no PESCAN por Silva et al. (2002) em uma comunidade de cerrado sentido restrito sobre Latossolo, localizado nas adjacências da área do presente estudo. Assim, a riqueza florística parece não ter sido influenciada pelo substrato sobre o qual a vegetação se desenvolve, mas sim pela proximidade geográfica entre áreas de formações savânicas de solos profundos que contribuem com a flora rupícola do cerrado rupestre (Pinto et al. 2009).

Os valores dos índices de diversidade foram próximos daqueles verificados em áreas de cerrado rupestre no Planalto Central (Amaral et al. 2006, Moura 2006, Pinto et al. 2009) e aos encontrados em áreas de cerrado sentido restrito em solos profundos (Felfili et al. 1994, 2001, Fonseca \& Silva Júnior 2004) (Tabela 2). As pequenas variações nos valores dos índices podem refletir uma maior abundância de algumas espécies mais tolerantes e adaptadas às condições extremas, em determinados sítios, principalmente edáficas, conforme sugerido por Ribeiro \& Walter (2008).

Das cinco famílias com maior número de espécies (Fabaceae, Myrtaceae, Vochysiaceae, Apocynaceae e Rubiaceae), as três primeiras são bem representadas em levantamentos florísticos conduzidos em áreas de cerrado rupestre (Amaral et al. 2006, Moura 2006, Felfili \& Fagg 2007, Pinto et al. 2009) e de cerrado sentido restrito sobre solos profundos (Felfili et al. 1994, 2001, Fonseca \& Silva Júnior 2004). Silva et al. (2002) verificaram que essas famílias, à exceção de Rubiaceae, também foram as mais ricas no cerrado sentido restrito amostradas sobre Latossolos no PESCAN. As famílias Asteraceae (3 espécies) e Melastomataceae (2), citadas como características de ambientes rupestres (Oliveira-Filho \& Fluminhan-Filho 1999,
Giulietti et al. 2000, Conceição \& Pirani 2005, Ribeiro \& Walter 2008), foram pouco representadas na área de estudo, provavelmente por se tratar de duas famílias cujas espécies possuem, em geral, hábito sub-arbustivo em áreas savânicas do Cerrado, normalmente com diâmetros inferiores ao adotado como critério de inclusão no presente estudo (DAS $\geq 5 \mathrm{~cm}$ ).

A maioria das espécies registradas no presente estudo é comum às áreas de cerrado sentido restrito sobre solos profundos e bem drenados localizadas no Brasil Central (Nunes et al. 2002, Ratter et al. 2003, Felfili et al. 2007). Além do mais, os maiores valores de similaridade de Czekanowski, foram registrados entre o cerrado rupestre do PESCAN e duas outras áreas de cerrado sobre solo profundo (Tabela 2). Desse modo, os resultados florísticos encontrados corroboram com as observações feitas por Eiten (1978), de que a vegetação existente nas encostas com afloramentos rochosos do PESCAN é composta por uma flora predominantemente acessória, caracterizada por elementos típicos do cerrado sentido restrito. No entanto, o cerrado rupestre do PESCAN apresentou espécies restritas a ambientes rupestres, como Schwartzia adamantium e Wunderlichia mirabilis (Mendonça et al. 2008, Ratter et al. 2000), bem como espécies pouco comuns no Cerrado, como Peltogyne confertiflora, Myrcia canescens e Myrcia variabilis (Ratter et al. 2003).

É importante destacar ainda que Schwartzia adamantium e Wunderlichia mirabilis contribuíram de forma expressiva na estrutura da vegetação estudada ( $4^{\circ}$ e $6^{\circ}$ VI, respectivamente). Juntas, essas duas espécies somam 7,59\% da densidade absoluta, 13,34\% da dominância absoluta e 9,53\% do VI total da comunidade. A importância estrutural dessas espécies confere ao cerrado rupestre do PESCAN, bem como de outras áreas de cerrado rupestre (Moura 2006, Pinto et al. 2009) característica florística e estrutural peculiar, quando comparado com as áreas de cerrado sentido restrito sobre solos profundos, nos quais não há registros destas espécies (Felfili et al. 1994, Fonsceca \& Silva Júnior 2004, Felfili et al. 2001). De acordo com Ribeiro \& Walter (2008), a identidade florística do cerrado rupestre se diferencia das demais fitofisionomias savânicas do bioma por apresentar espécies típicas e bem adaptadas a esses ambientes, afirmação essa corroborada pelos resultados encontrados para a vegetação arbustivo-arborea amostrada no PESCAN.

Outra característica relevante observada foi a elevada importância estrututural de Sclerolobium paniculatum (13\% do VI total) e Peltogyne 
confertiflora (7\% do VI total), que contribuíram, conjuntamente, com $21 \%$ de todos os indivíduos amostrados. Sclerolobium paniculatum é uma espécie comumente encontrada em áreas de cerrado sentido restrito sobre solos profundos (Nunes et al. 2002, Ratter et al. 2003), sendo também citada por Amaral et al. (2006) e Moura (2006) entre aquelas de maior VI em áreas de cerrado rupestre, podendo assim ser considerada como espécie generalista em relação ao substrato. De maneira distinta, Peltogyne confertiflora é considerada pouco freqüente em áreas de cerrado sentido restrito (Ratter et al. 2003), no entanto, parece estar bem adaptada ao ambiente rupestre do PESCAN, pois apresentou a maior densidade registrada na área (149 ind.ha ${ }^{-1}$ ).

Espécies que se desenvolvem sobre Neossolos Litólicos apresentam adaptações para sobreviver a variações extremas de temperatura e disponibilidade de água (Oliveira-Filho \& FuminhanFilho 1999). Dessa forma, é possível que as espécies com maiores valores de importância ou densidades como Schwartzia adamantium, Wunderlichia mirabilis, Sclerolobium paniculatum, Peltogyne confertiflora, Pterodon pubescens e Caryocar brasiliense apresentem sucesso em se estabelecer nestes locais, devido a sua tolerância às limitações físicas impostas pelo substrato rochoso.

Em termos estruturais, a área basal registrada foi alta $\left(12,39 \mathrm{~m}^{2} \cdot \mathrm{ha}^{-1}\right)$ quando comparada com os resultados encontrados em estudos realizados em áreas de cerrado sentido restrito sobre solos profundos no Brasil Central, que variou de 8,60 a 10,76 $\mathrm{m}^{2}$.ha $\mathrm{h}^{-1}$ e sobre Neossolos Litólicos, 3,67 a 11,03 $\mathrm{m}^{2}$.ha-1 (Tabela 2). A existência de afloramentos rochosos é considerada como limitante para o desenvolvimento da vegetação arbustivo-arbórea, contribuindo para o estabelecimento de comunidades estruturalmente mais abertas (Ribeiro \& Walter 1998). Essa característica foi observada em cerrado rupestre investigados por Amaral et al. (2006), Moura (2006) e Pinto et al. (2009). Entretanto, no presente estudo, verificou-se o contrário, pois o cerrado rupestre inventariado no PESCAN foi o que apresentou a maior área basal entre as áreas comparadas. Certamente isto se deve ao fato da elevada densidade (1.357 indivíduos.ha $\left.{ }^{-1}\right)$ e, em certos casos, ao grande porte de indivíduos de algumas espécies, como Sclerolobium paniculatum, Pterodon pubescens, Schwartzia adamantium, Caryocar brasiliensis e Wunderlichia mirabilis. Estes resultados sugerem a habilidade dessas espécies em explorar ambientes com condições físicas do substrato aparentemente limitantes.

A distribuição de frequência dos diâmetros do tipo "J-reverso" registrada para a vegetação arbustivo arbórea do PESCAN indica que a comunidade, caso não seja perturbada, poderá garantir o fluxo de indivíduos das primeiras para as últimas classes, mantendo assim a estrutura da comunidade, independente da composição de espécies, conforme também observado por Pinto \& Hay (2005). No entanto, nem todas as espécies alcançaram as maiores classes de diâmetro, uma vez que a maioria dos indivíduos e espécies é registrada nas primeiras classes de diâmetro, semelhante ao encontrado para as áreas de cerrado sentido restrito sobre solos profundos (Felfili et al. 1994, Felfili 2001). A maioria dos indivíduos com altura de até $4,2 \mathrm{~m}$, foi semelhante ao verificado por Amaral et al. (2006), Moura (2006) e Pinto et al. (2009), para áreas de cerrado rupestre.

A similaridade florística entre a área do presente estudo e as demais áreas usadas nas comparações foi baixa, uma vez que os índices de Czekanowski foram inferiores a 50\%. Ratter et al. (2003) verificaram que são poucas as espécies com ampla distribuição no bioma Cerrado e que uma parte expressiva apresenta distribuição restrita. Esse padrão de distribuição espacial das espécies ao longo do bioma pode ter contribuído para os baixos índices de similaridades encontrados. Entretanto, era esperado que a similaridade florística entre o PESCAN e as outras áreas de cerrado rupestre fosse maior, devido às condições ambientais semelhantes, principalmente em função da existência de Neossolos Litólicos, que poderiam atuar como fator de seleção para a existência de uma composição florística distinta e associada ao ambiente rupestre. O cerrado rupestre do PESCAN localiza-se em altitudes entre 800 e 1.000 m em relação ao nível do mar, enquanto as demais áreas comparadas se localizam no Planalto Central, em altitudes superiores a $1.000 \mathrm{~m}$. A altitude (Ratter et al. 2003) e as condições nutricionais dos solos (Eiten 1978, Ratter et al. 2000), também agem como fator de seleção das espécies no bioma Cerrado e devem ser incluídas em estudos futuros sobre a flora dos cerrados rupestres do bioma, para se entender as causas determinantes da riqueza, da composição florística e da estrutura da vegetação.

Os resultados desse estudo sugerem que a simples ocorrência de Neossolos Litólicos, supostamente limitantes ao desenvolvimento de algumas espécies arbustivo-arbóreas, não limitou o desenvolvimento da vegetação arbustivo-arbórea, nem condicionou o estabelecimento de uma flora diferenciada. Em termos estruturais, a área basal, a densidade e as distribuições diamétricas e de altura foram semelhantes e, às vezes, superiores a de cerrados sentido restrito sobre solos profundos do Planalto Central, confirmando as alegações de Ribeiro \& Walter (2008) de que cerrados rupestres podem apresentar estrutura que varia de cerrado ralo à cerrado típico. Em termos florísticos, foi observado que o cerrado rupestre do PESCAN abrigou tanto espécies com ampla distribuição em fisionomias sâvanicas do Cerrado, quanto espécies consideradas raras ou pouco comuns no bioma. Foi ainda observada elevada importância estrutural de espécies típicas e geralmente restritas e adaptadas a ambientes rupestres. Essas características estruturais e florísticas do cerrado rupestre estudado fazem do PESCAN uma importante área para a manutenção da composição e riqueza de espécies lenhosas da flora do bioma Cerrado. Além disso, como em áreas de cerrado rupestre o solo mostra-se inapto à agricultura, é de extrema importância o estudo e preservação destas áreas, uma vez que elas poderiam funcionar como fonte de propágulos para áreas vizinhas, contribuindo para recuperação de um bioma que hoje encontra-se extremamente fragmentado.

\section{Agradecimentos}

Agradecemos a Gabriel Damasco do Vale, Júlio César Sampaio, Raiana Quirino e Keila Sanches pela ajuda nos trabalhos de campo. A Carolyn Proença, Lúcia Helena Soares e Silva, Manoel Cláudio da Silva Júnior e Benedito Alísio da Silva Pereira pela ajuda na identificação do material botânico coletado. A Aloysio de Pádua Teixeira pela leitura prévia e sugestões ao manuscrito. À SEMARH de Goiás, em especial à administração do PESCAN, por autorizar a realização do estudo no Parque. À Fundação O Boticário de Proteção à Natureza pelo apoio financeiro ao projeto.

\section{Referências Bibliográficas}

Agência Ambiental de Goiás. <www3.agenciaambiental.go.gov.br/site/ conservacao/estadual.php> (último acesso em 22/01/2009).

AGP II - Angiosperm Phylogeny Group. 2003. An update of the angiosperm phylogeny group classification for the orders and families of flowering plants: APG II. Bot. J. Linn. Soc. 141(4):399-436.

AGUIAR, L.M.S., MACHADO, B.M. \& MARINHO-FILHO, J. 2004. A Diversidade Biológica do Cerrado. In Cerrado: ecologia e caracterização (L.M.S. Aguiar \& A.J.A Camargo, eds.). Embrapa Cerrados, Planaltina, p.17-40.

AMARAL, A.G., PEREIRA, F.F.O. \& MUNHOZ, C.B.R. 2006. Fitossociologia de uma área de cerrado rupestre na Fazenda Sucupira, Brasília-DF. Cerne 12(4):350-359.

BLOOM, S.A. 1981. Similarity indices in community studies: potential pitfalls. Mar. Ecol. Prog. Ser. 5:125-128.

CIENTEC - Fundação de Ciência e Tecnologia - Cientec. 2006. Mata nativa 2: manual do usuário. Cientec, Viçosa.

CONCEIÇÃO, A.A. \& PIRANI, J.R. 2005. Delimitação de habitats em campos rupestres na Chapada Diamantina: substratos, composição florística e aspectos estruturais. Bol. Bot. Univ. São Paulo. 23(1):85-111. 
COLWELL, R.K. 2006. Estimates: Statistical estimation of species richness and shared species from samples. Version 8.0. <http://viceroy.eeb.uconn. edu/estimates $>$ (último acesso em 07/10/2008).

CHAO, A. 1984. Nonparametric estimation of the number of classes in a population. Scand. J. Statist. 11:265-270.

EITEN, G. 1978. Delimitation of the cerrado concept. Vegetation 36(3):169-178.

FELFILI, J.M., FILGUEIRAS, T.S., HARIDASAN, M., SILVA Jr., M.C., MENDONÇA, R.C. \& REZENDE, A.V. 1994. Projeto Biogeografia do Bioma Cerrado: Vegetação \& Solos. Cad. Geoc. 12:75-166.

FELFILI, J.M. 2001. Distribuição de diâmetros de quatro áreas de cerrado sensu strictu na Chapada do Espigão Mestre do São Francisco. In Biogeografia do bioma cerrado: estudo fitofisionômico na Chapada do Espigão Mestre do São Francisco (J.M. Felfili \& M.C. Silva Jr., eds.). Universidade de Brasília, Brasília, DF, p.57-61.

FELFILI, J.M., SILVA Jr., M.C, SEVILHA, A.C., REZENDE, A.V., NOGUEIRA, P.E., WALTER, B. T., CHAGAS E SILVA, F. \& SALGADO, M.A.S. 2001. Fitossociologia da vegetação arbórea. In Biogeografia do bioma cerrado: estudo fitofisionômico na Chapada do Espigão Mestre do São Francisco (J.M. Felfili \& M.C. Silva Jr., eds.). Universidade de Brasília, Brasília, DF, p.35-56.

FELFILI, J.M., SOUZA-SILVA, J.C. \& SCARIOT, A. 2005. Biodiversidade, ecologia e conservação do cerrado: avanços no conhecimento. In Cerrado: ecologia, biodiversidade e conservação (A. Scariot, J.C. Souza-Silva \& J.M. Felfili, eds.). Ministério do Meio Ambiente, Brasília, DF, p.25-44.

FELFILI, J.M. \& FAGG, C.W. 2007. Floristic composition, diversity and structure of the "cerrado" sensu stricto on rocky soils in northern Goiás and southern Tocantins, Brazil. Rev. Brasil. Bot. 30(3):375-385.

FELFILI, J.M., REZENDE, A.V., SILVA Jr., M.C., SILVA, P.E.N., WALTER, B.M.T., IMAÑA-ENCINAS, J. \& SILVA, M.A. 2007. Fitossociologia da vegetação arbórea. In Biogeografia do Bioma Cerrado: Vegetação e Solos da Chapada dos Veadeiros (J.M. Felfili, A.V. Rezende \& M.C. Silva Jr., eds.) Editora Universidade de Brasília/Finatec, Brasília, DF, p.45-96.

FONSECA, M.S. \& SILVA Jr., M.C. 2004. Fitossociologia e similaridade florística entre trechos de Cerrado sentido restrito em interflúvio e em vale no Jardim Botânico de Brasília, DF. Acta Bot. Bras. 18(1):19-29.

GIULIETTI, A.M., HARLEY, R.M., QUEIROZ, L.P., WANDERLEY, M.G.L. \& PIRANI, J.R. 2000. Caracterização e endemismos nos campos rupestres da Cadeia do Espinhaço. In Tópicos Atuais em Botânica (T.B. Cavalcanti \& B.M.T. Walter, eds.). Embrapa Recursos Genéticos e Biotecnologia CENARGEN, Brasília, DF, p. 311-318.

HARLEY, R.M. 1995. Introduction. In Flora of the Pico das Almas, Chapada Diamantina, Brazil. (B.L. Stannard ed.). Royal Botanical Gardens, Kew, p.1-78.

KENT, M. \& COKER, P. 1992. Vegetation description and analysis: a practical approach. John Wiley \& Sons, London.

KLINK, C.A. \& MACHADO, R.B. 2005. Conservation of the Brazilian Cerrado. Conserv. Biol. 19(3):707-713.

MACHADO, R.B., RAMOS NETO, M.B., PEREIRA, P.G.P., CALDAS, E.F., GONÇALVES, D.A., SANTOS, N.S., TABOR, K. \& STEININGER, M. 2004. Estimativas da perda da área do Cerrado brasileiro. Conservação Internacional, Brasília, DF.

MARTINS, F.R. 1991. Estrutura de uma floresta mesófila. Editora Unicamp, Campinas.

MENDONÇA, R.C., FELFILI, J.M., WALTER, B.M.T., SILVA Jr., M.C., REZENDE, A.V., FILGUEIRAS, T.S., NOGUEIRA, P.E. \& FAGG, C.W. 2008. Flora Vascular do bioma Cerrado: checklist com 12.356 espécies. In Cerrado: ecologia e flora (S.M. Sano, S.P. Almeida \& J.F. Ribeiro, eds.). Embrapa Cerrados, Planaltina, p.421-1279.

MENDONÇA, R.C., FILGUEIRAS, T.S. \& FAGG, C.W. 2007. Análise Florística da Chapada dos Veadeiros. In Biogeografia do Bioma Cerrado: Vegetação e Solos da Chapada dos Veadeiros (J.M. Felfili, A.V. Rezende \& M.C. Silva Jr., eds.). Editora Universidade de Brasília/Finatec, Brasília, DF, p.119-237.

MITTERMEIER, R.A., ROBLES, P., HOFFMANN, M., PILGRIM, J., BROOKS, T., MITTERMEIER, C.G., LAMOREUX, J. \& FONSECA, G.B. 2005. Hotspots Revisited: earth's biologically richest and most endangered ecoregions. Conservation International, Mexico City.

MMA - Ministério do Meio Ambiente. 2007. Mapas de Cobertura dos Biomas Brasileiros. Ministério do Meio Ambiente, Secretaria de Nacional de Biodiversidade e Florestas, Brasília, DF.
MOURA, I. 2006. Fitossociologia de cerrado sensu stricto em afloramentos rochosos no Parque Estadual dos Pireneus, Pirenópolis, Goiás. Dissertação de Mestrado, Universidade Federal de Goiás, Goiânia.

MÜELLER-DOMBOIS, D. \& ELLENBERG, H. 1974. Aims and methods of vegetation ecology. John Wiley and Sons, New York.

MUNHOZ, C.B.R. \& PROENÇA, C.E.B. 1998. Composição florística do Município de Alto Paraíso de Goiás na Chapada dos Veadeiros. B. Herb. Ezechias Paulo Heringer 3:102-150.

NIMER, E. 1989. Climatologia do Brasil. 2 ed. IBGE, Rio de Janeiro.

NUNES, R.V., SILVA Jr., M.C., FELFILI, J.M. \& WALTER, B.M.T. 2002. Intervalos de classe para abundância, dominância e frequência do componente lenhoso do cerrado sentido restrito no Distrito Federal. R. Árvore 26(2):173-182.

OLIVEIRA-FILHO, A.T. 1994. Estudos ecológicos da vegetação como subsídios para programas de revegetação com espécies nativas: uma proposta metodológica. Cerne 1(1):64-72.

OLIVEIRA-FILHO, A.T. \& FLUMINHAN-FILHO, M. 1999. Ecologia da vegetação do Parque Florestal Quedas do Rio Bonito. Cerne 5(2):51-64.

PINTO, J.R.R. \& HAY, J.D.V. 2005. Mudanças florísticas e estruturais na comunidade arbórea de uma floresta de vale no Parque Nacional da Chapada dos Guimarães, Mato Grosso, Brasil. Rev. Brasil. Bot. 28(3):523-539.

PINTO, J.R.R., LENZA, E. \& PINTO, A. de S. 2009. Composição florística e estrutura da vegetação arbustivo-arbórea em um cerrado rupestre, Cocalzinho de Goiás, Goiás. Rev. Brasil. Bot. 32(1):23-32.

PIRANI, J.R., GIULIETTI, A.M. \& MELLO-SILVA, R. 1994. Checklist and patterns of geographic distribution of the vegetation of Serra do Ambrósio, Minas Gerais, Brazil. Rev. Brasil. Bot. 17(2):133-147.

RATTER, J.A., BRIDGEWATER, S., RIBEIRO, J.F., DIAS, T.A.B. \& SILVA, M.R. 2000. Estudo preliminar da distribuição das espécies lenhosas da fitofisionomia cerrado sentido restrito nos estados compreendidos pelo bioma cerrado. B. Herb. Ezechias Paulo Heringer 55-43.

RATTER, J.A., BRIDGEWATER, S. \& RIBEIRO, J.F. 2003. Analysis of the floristic composition of the Brazilian cerrado vegetation III: Comparison of the woody vegetation of 376 areas. Edinb. J. Bot. 60(1):57-109.

REATTO, A., CORREIA, J.R., SPERA, S.T. \& MARTINS, S. 1998. Solos do Bioma Cerrado: aspectos pedológicos. In Cerrado: ecologia e flora (S.M. Sano, S.P. Almeida \& J.F. Ribeiro, eds.). Embrapa Cerrados, Planaltina, p.108-149.

RIBEIRO, J.F. \& WALTER, B.M.T. 2008. As principais fitofisionomias do Bioma Cerrado. In Cerrado: ecologia e flora (S.M. Sano, S.P. Almeida \& J.F. Ribeiro, eds.). Embrapa Cerrados, Planaltina. p.151 -212.

ROMERO, R. \& NAKAJIMA, J.N. 1999. Espécies endêmicas do Parque Nacional da Serra da Canastra. Rev. Brasil. Bot. 22(2):259-265.

ROMERO, R. \& MARTINS, A.B. 2002. Melastomataceae do Parque Nacional da Serra da Canastra, Minas Gerais, Brasil. Rev. Brasil. Bot. 25(1):19-24.

SANTOS, A.J. 2003. Estimativas de riqueza em espécies. In: Métodos de estudos em biologia da conservação e manejo da vida silvestre. (L. Cullen Jr., R. Rudran \& C. Valladares-Padua, org.). Editora UFPR/, Fundação o Boticário de Proteção a Natureza, Curitiba. p.19-41.

SCOLFORO, J.R.S. 1993. Inventário florestal. Escola Superior de Agricultura de Lavras, Lavras.

SILVA, L.O., COSTA, D.A., SANTO FILHO, K.E., FERREIRA, H.D. \& BRANDÃO, D. 2002. Levantamento fitossociológico em duas áreas de cerrado sensu strictu no Parque Estadual da Serra de Caldas Novas, Goiás. Acta Bot. Bras. 16(1):43-53.

SIMON, M.F. \& PROENÇA, C. 2000. Phytogeographic patters of Mimosa (Mimosoidea, Leguminosae) in the Cerrado biome of Brazil: an indicator genus of high-altitude centers of endemism? Biol. Conserv. 96(3):279-296.

VITTA, F.A. 2002. Diversidade e conservação da flora nos campos rupestres da Cadeia do Espinhaço em Minas Gerais. In Biodiversidade, conservação e uso sustentável da flora do Brasil (E.L. Araújo, A.N. Moura; E.S.B. Sampaio, L.M.S. Gestinari, \& J.M.T. Carneiro, eds.). Imprensa Universitária - UFRPE, Recife, p.90-94.

WALTER, B.M.T. 2006. Fitofisionomias do bioma Cerrado: síntese terminológica e relações florísticas. Tese de Doutorado, Universidade de Brasília, Brasília.

Recebido em 07/04/09

Versão reformulada recebida em 07/06/10

Publicado em 21/06/10 